INPLASY

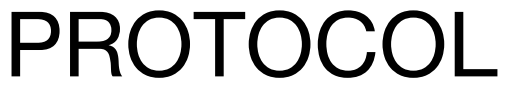

To cite: Sun et al. Efficacy and safety of Traditional Chinese patent medicine on carotid artery atherosclerosis in adults: a network meta-analysis protocol. Inplasy protocol 2020120036. doi:

10.37766/inplasy2020.12.0036

Received: 06 December 2020

Published: 06 December 2020

Corresponding author:

Shichuan Shao

sdstastsq@163.com

Author Affiliation:

Tai'an City Central Hospital

Support: No funding to disclose.

Review Stage at time of this submission: The review has not yet started.

Conflicts of interest:

The authors have no conflicts of interest to disclose.

\section{Efficacy and safety of Traditional Chinese patent medicine on carotid artery atherosclerosis in adults: a network meta-analysis protocol}

Sun, HQ17 Qu, W2; Chen, GJ3; Sun, XN4; Shao, SC5.

Review question / Objective: Numerous studies have indicated that the combination treatment with TCPM and western medicine generate better efficacy than western medicine alone.However, studies comparing the efficacy and safety of different TCPM on adult AS are currently lacking. Information sources: Electronic research will be organized in PubMed, Cochrane Library, Embase, Web of Science, Chinese BioMedical Literature Database, China National Knowledge Infrastructure Database,China Science and Technology Journal Database and Wanfang Database. The search time limite from inception until October 2020. There is no languages and publication date restrictions. As a supplement to the study, the references in the included literature will be manually retrieved.

INPLASY registration number: This protocol was registered with the International Platform of Registered Systematic Review and Meta-Analysis Protocols (INPLASY) on 06 December 2020 and was last updated on 06 December 2020 (registration number INPLASY2020120036).

\section{INTRODUCTION}

Review question / Objective: Numerous studies have indicated that the combination treatment with TCPM and western medicine generate better efficacy than western medicine alone.However, studies comparing the efficacy and safety of different TCPM on adult AS are currently lacking.

Condition being studied: Electronic research will be organized in PubMed, Cochrane Library, Embase, Web of Science, 
Chinese BioMedical Literature Database, China National Knowledge Infrastructure Database,China Science and Technology Journal Database and Wanfang Database. The search time limite from inception until October 2020. There is no languages and publication date restrictions. As a supplement to the study, the references in the included literature will be manually retrieved.

\section{METHODS}

Participant or population: Patients are diagnosed with aortic atherosclerosis by vascular colour doppler ultrasonography. The diagnostic criteria for carotid artery plaque is defined as carotid artery intimamedia thickness (IMT) $\geq 1.5 \mathrm{~mm}$, which indicates plaque formation. Age $\geq 18$, regardless of gender or race.

Intervention: The control group received western medicine treatment, including lipid-lowering, antiplatelet aggregation, and other underlying diseases therapy. The experimental group receive the same western medicine treatment plus a TCPM for AS. Any mode of administration of TCPM will be included, such as oral and intravenous administration. There is no limit on the dosage and duration of intervention for TCPM.

Comparator: The control group received western medicine treatment, including lipid-lowering, antiplatelet aggregation, and other underlying diseases therapy.

Study designs to be included: The study included in the NMA is randomized controlled trials(RCTs) for adult AS.

\section{Eligibility criteria: (1) Study design The} study included in the NMA is randomized controlled trials (RCTs) for adult AS. (2) Participants Patients are diagnosed with aortic atherosclerosis by vascular colour doppler ultrasonography. The diagnostic criteria for carotid artery plaque is defined as carotid artery intima-media thickness (IMT) $\geq 1.5 \mathrm{~mm}$, which indicates plaque formation. Age $\geq 18$, regardless of gender or race. (3) Interventions The control group received western medicine treatment, including lipid-lowering, antiplatelet aggregation, and other underlying diseases therapy. The experimental group receive the same western medicine treatment plus a TCPM for AS. Any mode of administration of TCPM will be included, such as oral and intravenous administration. There is no limit on the dosage and duration of intervention for TCPM. (4) Outcomes Primary outcome indicators: IMT, total carotid plaque area (TPA), Crouse plaque score, the incidence of adverse reactions. Secondary outcome indicators: TC, triacylglycerol (TG), LDL-C, HDL-C levels.

Information sources: Electronic research will be organized in PubMed, Cochrane Library, Embase, Web of Science, Chinese BioMedical Literature Database, China National Knowledge Infrastructure Database,China Science and Technology Journal Database and Wanfang Database. The search time limite from inception until October 2020. There is no languages and publication date restrictions. As a supplement to the study, the references in the included literature will be manually retrieved.

Main outcome(s): Primary outcome indicators: IMT, total carotid plaque area (TPA), Crouse plaque score, the incidence of adverse reactions. Secondary outcome indicators: TC, triacylglycerol (TG),LDLC,HDL-C levels.

Quality assessment / Risk of bias analysis: Collaboration's risk of bias tool(Cochrane ROB) will assess the risk of bias [23].The instrument Cochrane ROB contains the following six items: (1)selection bias;(2) performance bias;(3) detection bias;(4) attrition bias;(5) reporting bias; (6) other potential bias.Risk of bias will be evaluated by three grades:(1) low bias risk;(2) uncertainty of bias risk;(3) high bias risk. Two reviewers will independently conduct the risk of bias assessment.Any disagreement will be adjudicated by a third researcher. 
Strategy of data synthesis: If the outcome is dichotomic variables, odds ratios(ORs) will given with the $95 \%$ confidence interval (Cl). If the outcome is continuous variables, standardised mean differences (SMD) will given with the $95 \% \mathrm{Cl}$. Winbugs 1.4 .3 software will be used to fit the Bayesian hierarchical model.Three Markov chain Monte Carlo (MCMC) will run with 50,000 iterations and a burn-in period of 20,000 . The network relationship graph will be plotted by using STATA15.0 software.The thicker the line, the higher the number of RCTs compared between the two interventions. The bigger the dot, the larger the sample size of the intervention. Surface under the cumulative ranking area (SUCRA) will be calculated. The larger the SUCRA values, the better the efficacy of interventions.

Subgroup analysis: As for the subgroup analysis, we will analyze the gender, age, treatment period and blood lipid to explore the influence of these factors on the total effect.

Sensibility analysis: As for the subgroup analysis, we will analyze the gender, age, treatment period and blood lipid to explore the influence of these factors on the total effect.

\section{Country(ies) involved: China.}

Keywords: traditional Chinese patent medicine, atherosclerosis, protocol, systematic review.

Contributions of each author:

Author 1 - Huiqing Sun - The author drafted the manuscript.

Author 2 - Wei Qu - The author provided statistical expertise.

Author 3 - Guangjia Chen - The author contributed to the development of the selection criteria, and the risk of bias assessment strategy.

Author 4 - Xiaonan Sun - The author read, provided feedback and approved the final manuscript.
Author 5 - Shichuan Shao - The author read, provided feedback and approved the final manuscript. 